\title{
Mediterranean alcohol-drinking pattern and mortality in the SUN (Seguimiento Universidad de Navarra) Project: a prospective cohort study
}

\author{
Alfredo Gea ${ }^{1}$, Maira Bes-Rastrollo ${ }^{1,2}$, Estefania Toledo ${ }^{1,2}$, Martin Garcia-Lopez ${ }^{3}$, Juan J. Beunza ${ }^{1,4}$, \\ Ramon Estruch $^{2,5}$ and Miguel A. Martinez-Gonzalez ${ }^{1,2 *}$ \\ ${ }^{1}$ Department of Preventive Medicine and Public Health, School of Medicine-Clinica, University of Navarra, \\ Irunlarrea 1, Pamplona 31008, Navarra, Spain \\ ${ }^{2}$ CIBER Fisiopatología de la Obesidad y Nutrición (CIBERObn), Instituto de Salud Carlos III, Madrid, Spain \\ ${ }^{3}$ Department of Cardiology, Clinica University of Navarra, Pamplona, Spain \\ ${ }^{4}$ School of Medicine, Universidad Europea de Madrid, Madrid, Spain \\ ${ }^{5}$ Department of Internal Medicine, Hospital Clinic, University of Barcelona, Barcelona, Spain \\ (Submitted 2 September 2013 - Final revision received 25 November 2013 - Accepted 13 December 2013 - First published online 30 January 2014)
}

\section{Abstract}

Moderate alcohol intake has been related to lower mortality. However, alcohol use includes other dimensions beyond the amount of alcohol consumed. These aspects have not been sufficiently studied as a comprehensive entity. We aimed to test the relationship between an overall alcohol-drinking pattern and all-cause mortality. In a Mediterranean cohort study, we followed 18394 Spanish participants up to 12 years. A validated 136-item FFQ was used to assess baseline alcohol intake. We developed a score assessing simultaneously seven aspects of alcohol consumption to capture the conformity to a traditional Mediterranean alcohol-drinking pattern (MADP). It positively scored moderate alcohol intake, alcohol intake spread out over the week, low spirit consumption, wine preference, red wine consumption, wine consumed during meals and avoidance of binge drinking. During the follow-up, 206 deaths were identified. For each 2-point increment in a 0-9 score of adherence to the MADP, we observed a $25 \%$ relative risk reduction in mortality (95\% CI $11,38 \%$ ). Within each category of alcohol intake, a higher adherence to the MADP was associated with lower mortality. Abstainers (excluded from the calculations of the MADP) exhibited higher mortality (hazard ratio 1.82, 95\% CI 1.14, 2.90) than participants highly adherent to the MADP. In conclusion, better adherence to an overall healthy alcohol-drinking pattern was associated with reduced mortality when compared with abstention or departure from this pattern. This reduction goes beyond the inverse association usually observed for moderate alcohol drinking. Even moderate drinkers can benefit from the advice to follow a traditional MADP.

Key words: Drinking patterns: Alcohol: Mortality: Cohort studies

A U-shaped association between alcohol intake and the risk of cardiovascular ${ }^{(1)}$, cancer $^{(2)}$ and non-cardiovascularnon-cancer mortality ${ }^{(3)}$ has frequently been reported. The pathological effects of excess alcohol consumption have been widely studied in many different tissues such as the myocardium $^{(4,5)}$. Moreover, deleterious effects of heavy drinking have also been reported in epidemiological studies ${ }^{(6)}$. Low-to-moderate alcohol intake when compared with abstention or heavy drinking is usually assumed to reduce the risk of major chronic disease and all-cause mortality. The mechanisms proposed to explain this inverse association with moderate alcohol intake include increases in serum HDLcholesterol, inhibition of platelet production, activation and aggregation, increased fibrinolysis ${ }^{(7)}$, beneficial effects on endothelial function and inflammation ${ }^{(8)}$, and enhanced insulin sensitivity ${ }^{(9)}$. However, drinking alcoholic beverages involves other dimensions beyond the precise amount of alcohol intake. For example, alcohol intake can be conceptualised as an element that forms part of an overall dietary pattern or as a substance consumed in order to seek psychoactive effects. In the culinary tradition of Mediterranean countries, alcohol intake used to be moderate, spread out over the week, preferably from wine, consumed with meals and without excess ${ }^{(10)}$, contrary to the binge-drinking pattern of concentrated consumption of spirits ${ }^{(11)}$. Our hypothesis was that a high adherence to an overall healthy alcohol-drinking pattern involving many dimensions of drinking behaviour can reduce all-cause mortality, beyond the reduction observed for

Abbreviations: HR, hazard ratio; MADP, Mediterranean alcohol-drinking pattern; SUN, Seguimiento Universidad de Navarra.

*Corresponding author: Professor M. A. Martinez-Gonzalez, email mamartinez@unav.es 
moderate alcohol intake. In this context, we developed a score to capture the conformity to the traditional Mediterranean alcohol-drinking pattern (MADP) and tested its relationship with the risk of all-cause mortality.

\section{Methods}

The 'Seguimiento Universidad de Navarra' (University of Navarra Follow-up Study) (SUN) Project is a dynamic and multi-purpose cohort study entirely composed of highly educated subjects (university graduates). Up to September 2009, 20335 subjects answered the baseline questionnaire. Information was updated biennially. For the present analysis, 404 participants with total daily energy intake out of percentiles 1 and 99 were excluded. Among the remaining 19931 participants, 18394 were successfully followed up (overall retention rate $92.3 \%$; Fig. 1). The present study was approved by the Institutional Review Board of the University of Navarra. Details of the design and methods of this cohort study have been described elsewhere ${ }^{(12)}$.

\section{Mediterranean alcohol-drinking pattern}

Alcoholic beverage consumption (red wine, non-red wine, beer and spirits) and other information about alcohol-drinking habits during the year preceding enrolment were gathered in the baseline assessment, including a validated 136-item semiquantitative $\mathrm{FFQ}^{(13)}$

A 0- to 9-point score was developed to capture the conformity to the traditional MADP. We scored seven items (Table 1): (1) moderate total alcohol intake - positively scored with 2 points if the consumption was $10-50 \mathrm{~g} / \mathrm{d}$ (men) or $5-25 \mathrm{~g} / \mathrm{d}$ (women) ${ }^{(14)}$; intakes below this range were assigned 1 point and intakes above this range were assigned 0 points; (2) alcohol intake spread out over the week - (i.e. the total weekly amount of alcohol ingested was evenly distributed across the days of the week) we computed the ratio between the number of drinking days per week and total $\mathrm{g} /$ week of alcohol intake and categorised it in quartiles; we assigned 2 points to participants in the highest quartile (most days in a week and lower total amount), 1 point to those in the second and third quartiles and 0 points in the lowest quartile (few days and high amount); (3) low consumption of spirits - if the proportion of alcohol

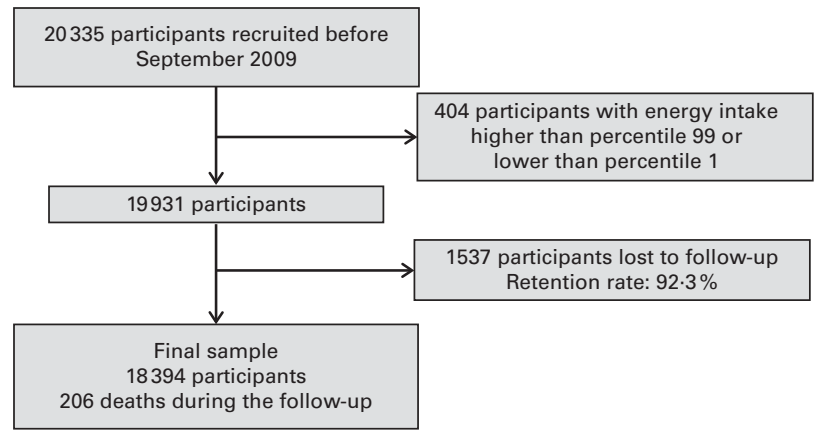

Fig. 1. Flow chart of the study participants in the Seguimiento Universidad de Navarra (SUN) Project 1999-2012. from spirits was lower than $25 \%$, participants received 1 point; (4) preference for wine - we scored 1 point if the proportion of alcohol derived from wine was at least $75 \%$ of total alcohol intake; (5) consuming wine preferentially during meals - we scored 1 point if the proportion of alcohol from wine consumed with meals was at least $75 \%$ of total alcohol from wine consumed; (6) preference for red wine over other types of wine we scored 1 point if the proportion of red wine consumed is at least $75 \%$ of total wine consumption; (7) avoidance of excess drinking occasions - we assigned 1 point if the maximum number of drinks consumed in a single weekday, or a day during the weekend, or in special occasions never exceeded five drinks.

We selected these cut-off points in order to better capture the traditional Mediterranean drinking pattern as described in previous publications ${ }^{(14-16)}$, and also taking into account the shape of the dose-response curve observed in restricted cubic spline analyses for continuous variables.

Once these seven items are summed, the MADP score could potentially range from 0 to 9 points. We grouped participants into four categories according to this score: 0 to 2 points (low adherence); 3 to 4 (moderate-low); 5 to 6 (moderate-high); 7 to 9 (high adherence). Abstainers, who did not drink at all, were excluded from this computation, and they were classified in a fifth group.

\section{Mortality assessment}

Participants in the cohort study were carefully followed up in order to detect each death. At least annually, participants were contacted by mail and asked about changes of postal address. If postal mail failed, we used telephone or email contacts. We also exchanged information with the alumni associations and other professional associations to track participants. The closest relative, the appropriate professional association and the postal system allowed us to identify more than $85 \%$ of deaths. For the rest of the deaths, we checked the National Death Index every 6 months to confirm the vital status of all our participants with no updated information. Death certificates and medical records of deceased participants were obtained. Causes of death were adjudicated, blinded to alcohol or dietary information, according to the International Classification of Diseases, 10th Revision ${ }^{(17)}$, and grouped as cardiovascular, cancer and other causes.

\section{Covariate assessment}

We gathered information about anthropometric, sociodemographic, lifestyle and medical variables from the baseline questionnaire. Physical activity was assessed through a validated questionnaire ${ }^{(18)}$. Adherence to the Mediterranean dietary pattern was evaluated using a well-known score ${ }^{(14)}$; however, we excluded alcohol intake to avoid overlapping with our main exposure.

\section{Statistical analysis}

To assess the relationship between adherence to the MADP and the risk of mortality, Cox regression models were fitted. 
Table 1. Score of the Mediterranean alcohol-drinking pattern

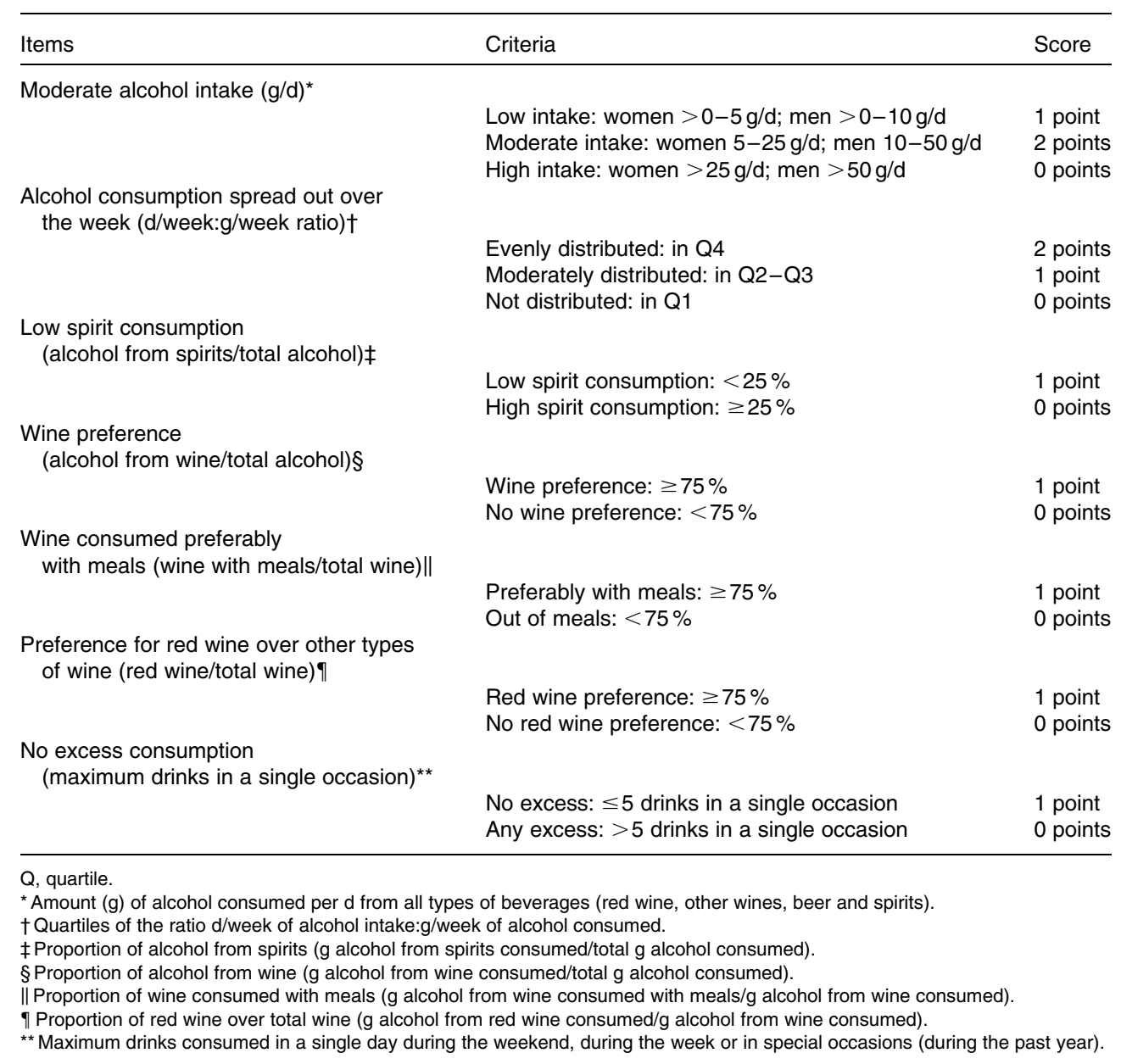

Hazard ratios (HR) and their 95\% CI were calculated using the high-adherence group as the reference category. We also computed the HR for abstainers using this same category as the reference group. For the Cox regression model, age was the underlying time variable and exit time was defined as date of death or date when completing the last follow-up questionnaire (for survivors).

We also evaluated the risk of mortality associated with a 2-point increment in the MADP score, by introducing in the model the total score divided by 2 as a continuous variable. Tests for linear trend were also conducted. For these two latter analyses, abstainers were excluded.

First, age and sex were introduced in the model, and then, in the multiple-adjusted model, we additionally considered as potential confounders BMI $\left(\mathrm{kg} / \mathrm{m}^{2}\right)$, total energy intake $(\mathrm{kJ} / \mathrm{d})$, physical activity (metabolic equivalent task-h/week), prevalent or previous hypertension, prevalent hypercholesterolaemia, smoking habit (current smoker, former smoker or never smoker), tertiles of adherence to the Mediterranean dietary pattern, prevalent or previous cancer, presence of diabetes or CVD at baseline, and time spent watching television (h/week).

To assess the individual contribution of every specific MADP item, we fitted Cox regression models for each of the seven items using the highest score as the reference category. We evaluated the influence of each single item on mortality rates by alternately subtracting one single component from the overall MADP score, following the methodology of a previous article ${ }^{(19)}$.

We conducted sensitivity analyses by rerunning all the models under different assumptions.

To compare the baseline characteristics of the participants according to the adherence to the MADP, we performed ANOVA (for continuous variables) and $\chi^{2}$ (for categorical variables) tests. Analyses were performed using STATA version 12.0 (StataCorp).

\section{Results}

During the follow-up period (137479 person-years), a total of 206 deaths were identified. The main characteristics of the 18394 participants categorised according to their adherence to the MADP are displayed in Table 2.

Participants with a higher adherence to the MADP were older ( $46 \cdot 0$ years), less physically active, were less likely to be current smokers but more prone to be former smokers, and had better average adherence to the Mediterranean dietary pattern.

An inverse association between the MADP as a continuous variable and the risk of mortality was apparent (HR 0.75 , $95 \%$ CI $0.62,0.89$ for every 2-point increase in the MADP), with a significant inverse linear trend $(P=0.003)$. 
Table 2. Baseline characteristics according to the categories of the Mediterranean alcohol-drinking pattern in the Seguimiento Universidad de Navarra Project 1999-2012

(Mean values and standard deviations or percentages)

\begin{tabular}{|c|c|c|c|c|c|c|c|c|c|c|c|}
\hline & \multicolumn{11}{|c|}{ Mediterranean alcohol-drinking pattern } \\
\hline & \multicolumn{2}{|c|}{ Abstainers } & \multicolumn{2}{|c|}{ Low $(0-2)$} & \multicolumn{2}{|c|}{$\begin{array}{l}\text { Moderate-low } \\
\quad(3-4)\end{array}$} & \multicolumn{2}{|c|}{$\begin{array}{l}\text { Moderate-high } \\
\quad(5-6)\end{array}$} & \multicolumn{2}{|c|}{ High (7-9) } & \multirow[b]{2}{*}{$P$} \\
\hline & Mean & SD & Mean & SD & Mean & SD & Mean & SD & Mean & SD & \\
\hline$n$ & \multicolumn{2}{|c|}{3240} & \multicolumn{2}{|c|}{1469} & \multicolumn{2}{|c|}{5401} & \multicolumn{2}{|c|}{5146} & \multicolumn{2}{|c|}{3138} & \\
\hline Alcohol intake $(\mathrm{g} / \mathrm{d})$ & \multicolumn{2}{|c|}{-} & $10 \cdot 2$ & 21.5 & 7.9 & 11.5 & $10 \cdot 1$ & $10 \cdot 3$ & $11 \cdot 1$ & $9 \cdot 0$ & $<0.001$ \\
\hline Wine consumption ( $\mathrm{g}$ alcohol/d) & \multicolumn{2}{|c|}{-} & $2 \cdot 7$ & $10 \cdot 1$ & $2 \cdot 5$ & $7 \cdot 2$ & 4.9 & $7 \cdot 3$ & 8.6 & 7.9 & $<0.001$ \\
\hline Red wine consumption ( $\mathrm{g}$ alcohol/d) & \multicolumn{2}{|c|}{-} & 1.5 & $6 \cdot 3$ & 1.5 & $5 \cdot 2$ & 3.1 & $5 \cdot 7$ & $6 \cdot 0$ & $7 \cdot 2$ & $<0.001$ \\
\hline Spirit consumption (g alcohol/d) & \multicolumn{2}{|c|}{ _- } & 3.7 & $8 \cdot 3$ & $2 \cdot 0$ & 3.5 & $1 \cdot 1$ & $2 \cdot 3$ & 0.4 & 0.9 & $<0.001$ \\
\hline Beer consumption ( $\mathrm{g}$ alcohol/d) & \multicolumn{2}{|c|}{ - } & $3 \cdot 8$ & $10 \cdot 7$ & $3 \cdot 2$ & $5 \cdot 8$ & $4 \cdot 0$ & $5 \cdot 3$ & $2 \cdot 0$ & 3.5 & $<0.001$ \\
\hline Ratio (d/week:g/week) & \multicolumn{2}{|c|}{ _- } & 0.09 & 0.13 & 0.27 & 0.38 & 0.47 & 0.68 & 0.73 & 0.95 & $<0.001$ \\
\hline $\begin{array}{l}\text { More than five drinks in } \\
\text { a single occasion (\%) }\end{array}$ & \multicolumn{2}{|c|}{-} & \multicolumn{2}{|c|}{68.0} & \multicolumn{2}{|c|}{43.7} & \multicolumn{2}{|c|}{30.2} & \multicolumn{2}{|c|}{$7 \cdot 1$} & $<0.001$ \\
\hline $\begin{array}{l}\text { Proportion of wine consumed } \\
\text { with meals (\%) }\end{array}$ & \multicolumn{2}{|c|}{-} & 8 & 21 & 18 & 32 & 51 & 45 & 85 & 31 & $<0.001$ \\
\hline Sex, women $(\%)$ & \multicolumn{2}{|c|}{$80 \cdot 3$} & \multicolumn{2}{|c|}{$59 \cdot 8$} & & & 5 & & & & $<0.001$ \\
\hline Age (years) & 36.8 & 11.9 & $31 \cdot 1$ & $9 \cdot 7$ & 34.5 & $11 \cdot 0$ & 41.4 & $11 \cdot 7$ & $46 \cdot 0$ & 11.4 & $<0.001$ \\
\hline BMI $\left(\mathrm{kg} / \mathrm{m}^{2}\right)$ & $22 \cdot 9$ & 3.6 & 23.3 & 3.7 & 23.3 & 3.5 & 23.9 & 3.5 & $24 \cdot 0$ & 3.3 & $<0.001$ \\
\hline Physical activity (MET-h/week) & $20 \cdot 3$ & 24.5 & $20 \cdot 6$ & $21 \cdot 8$ & $21 \cdot 7$ & 22.5 & $22 \cdot 0$ & $22 \cdot 4$ & 21.6 & $21 \cdot 3$ & 0.005 \\
\hline Time spent watching television ( $\mathrm{h} /$ week) & $11 \cdot 3$ & $9 \cdot 1$ & $13 \cdot 2$ & $9 \cdot 7$ & 11.9 & 8.7 & $11 \cdot 2$ & $8 \cdot 0$ & $10 \cdot 1$ & $8 \cdot 1$ & $<0.001$ \\
\hline Current smokers (\%) & & & & & & & 2 & & & & $<0.001$ \\
\hline Former smokers (\%) & & & & & & & 3 & & & & \\
\hline Prevalent hypertension (\%) & 6 & & & & & & 8 & & & & $<0.001$ \\
\hline Prevalent hypercholesterolaemia (\%) & 1 & & & & & & & & & & $<0.001$ \\
\hline Prevalent or previous cancer (\%) & 4 & & & & & & & & & & $<0.001$ \\
\hline Prevalent type 2 diabetes mellitus (\%) & & & & & & & & & & & $<0.001$ \\
\hline Prevalent or previous CVD (\%) & & & & & & & 1 & & & & $<0.001$ \\
\hline Total energy intake & & & & & & & & & & & $<0.001$ \\
\hline $\mathrm{kcal} / \mathrm{d}$ & 2487 & 801 & 2619 & 825 & 2534 & 793 & 2499 & 766 & 2470 & 758 & \\
\hline $\mathrm{kJ} / \mathrm{d}$ & 10406 & 3354 & 10958 & 3452 & 10603 & 3318 & 10456 & 3205 & 10335 & 3172 & \\
\hline Carbohydrates (\% of total energy intake) & $45 \cdot 3$ & $7 \cdot 8$ & $42 \cdot 6$ & $7 \cdot 6$ & $42 \cdot 9$ & $7 \cdot 1$ & $43 \cdot 1$ & $7 \cdot 5$ & 43.6 & $7 \cdot 3$ & $<0.001$ \\
\hline Proteins ( $\%$ of total energy intake) & $18 \cdot 3$ & 3.5 & $17 \cdot 6$ & 3.3 & $17 \cdot 9$ & 3.2 & $18 \cdot 0$ & 3.2 & $18 \cdot 0$ & 3.3 & $<0.001$ \\
\hline Lipids (\% of total energy intake) & $36 \cdot 5$ & $7 \cdot 1$ & $37 \cdot 2$ & $6 \cdot 9$ & 37.1 & $6 \cdot 3$ & $36 \cdot 3$ & 6.5 & 35.8 & $6 \cdot 4$ & $<0.001$ \\
\hline SFA (\% of total energy intake) & $12 \cdot 5$ & $3 \cdot 6$ & $12 \cdot 8$ & 3.3 & $12 \cdot 7$ & $3 \cdot 1$ & $12 \cdot 3$ & $3 \cdot 2$ & $12 \cdot 2$ & $3 \cdot 2$ & $<0.001$ \\
\hline MUFA (\% of total energy intake) & $15 \cdot 4$ & 3.9 & $15 \cdot 7$ & 3.7 & $15 \cdot 8$ & 3.5 & $15 \cdot 7$ & 3.7 & $15 \cdot 6$ & 3.8 & 0.002 \\
\hline PUFA ( $\%$ of total energy intake) & $5 \cdot 2$ & $1 \cdot 7$ & 5.4 & $1 \cdot 7$ & $5 \cdot 3$ & 1.6 & $5 \cdot 2$ & 1.5 & $5 \cdot 1$ & 1.5 & $<0.001$ \\
\hline Total fibre intake $(\mathrm{g} / \mathrm{d})$ & $30 \cdot 3$ & $15 \cdot 4$ & $27 \cdot 0$ & $12 \cdot 8$ & $28 \cdot 0$ & $13 \cdot 4$ & $29 \cdot 0$ & 13.7 & $30 \cdot 2$ & $14 \cdot 2$ & $<0.001$ \\
\hline Mediterranean dietary pattern & 3.9 & 1.8 & 3.7 & $1 \cdot 7$ & 3.8 & 1.7 & $4 \cdot 1$ & 1.7 & 4.2 & $1 \cdot 7$ & $<0.001$ \\
\hline
\end{tabular}

MET, metabolic equivalent task.

As shown in Fig. 2, the inverse association between the MADP and the risk of mortality remained apparent within the categories of total alcohol intake, suggesting that additional reductions in mortality were associated with better adherence to the MADP beyond the effect of moderate alcohol intake.

Moderate alcohol intake $(5-25 \mathrm{~g} / \mathrm{d}$ in women and $10-50 \mathrm{~g} / \mathrm{d}$ in men) was inversely associated with all-cause mortality compared with abstainers in the present sample, but the $\mathrm{CI}$ included the null value (HR 0.69, $95 \%$ CI 0.45, 1.06). However, beyond these results, adherence to the overall MADP in the highest level ( 7 to 9 points) showed a stronger reduction in the risk of mortality $v$. abstainers that was statistically significant (HR 0.55, $95 \%$ CI 0.35, 0.88; $P=0.012$ ).

When we used the highest category of adherence ( 7 to 9 points) to the MADP as the reference category, participants with a low adherence to the MADP (HR 3.09, 95\% CI 1.74, 5.50) and abstainers (HR 1.82, 95\% CI 1.14, 2.90) had a significantly higher risk of death than those with the highest adherence to the MADP, after adjustment for relevant confounders (Table 3). Participants with a moderate-low and moderate-high adherence also have a higher risk of death, but not statistically significant. Further adjustment for educational levels did not lead to any substantial change in these estimates.

The association between each single component of the MADP and the risk of mortality is presented in Table 4. After additional adjustment for all the other items, every MADP component was individually associated with a lower risk of death in its point estimate (data not shown), but usually the CI included the null value.

Every single component of the MADP seemed to have a similar influence on the inverse association with the risk of mortality, except for the total amount of alcohol consumed, the influence of which was slightly higher.

The main results of the present study were consistent for almost all the different scenarios that we included in the sensitivity analyses. For each additional 2-point increment in the MADP, we found stronger reductions for cardiovascular deaths (HR 0.55, 95\% CI 0.35, 0.84) than for cancer deaths 


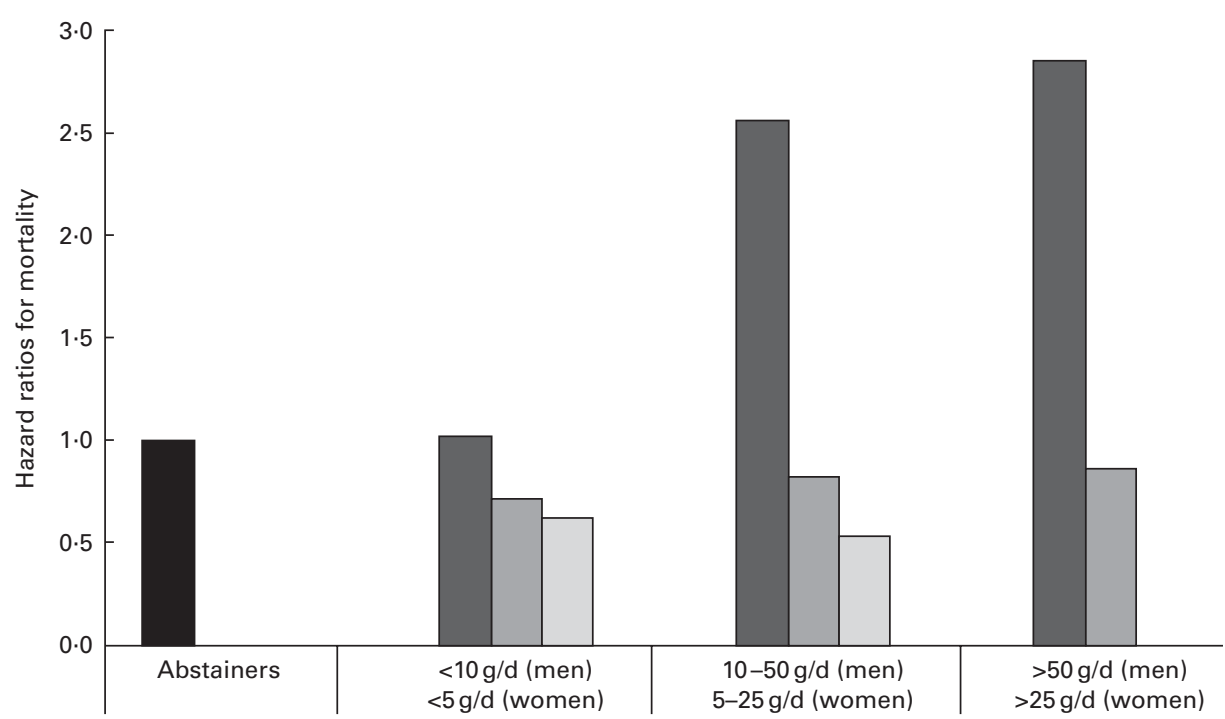

Alcohol intake

Fig. 2. Hazard ratios for mortality according to the categories of the Mediterranean alcohol-drinking pattern (MADP) within each category of alcohol intake in the Seguimiento Universidad de Navarra (SUN) Project 1999-2012. \, Low MADP (0-2); $\square$, moderate MADP (3-6); $\square$, high MADP (7-9). Adjusted for age, sex, BMI $\left(\mathrm{kg} / \mathrm{m}^{2}\right)$, total energy intake $(\mathrm{kJ} / \mathrm{d})$, physical activity (metabolic equivalent task-h/week), prevalent hypertension, prevalent hypercholesterolaemia, smoking habit (current smoker, former smoker or never smoker), Mediterranean dietary pattern (tertiles of adherence), prevalent or previous cancer, diabetes or CVD, and watching television (h/week).

(HR 0.86, 95\% CI $0.65,1 \cdot 13$ ). To rule out the possible confounding effect of smoking habit, we reran the analysis only among never smokers, and we obtained the results in the same direction but without statistical significance due to the small number of deaths occurred among never smokers.

\section{Discussion}

To our knowledge, this is the first study assessing an overall alcohol-drinking pattern to comprehensively account for different aspects of alcohol consumption beyond the total amount of alcohol intake. Consistent with previous studies ${ }^{(1-3)}$, we observed lower mortality among moderate drinkers. However, with our novel approach, we found that participants with a high adherence to a traditional Mediterranean alcohol-drinking pattern exhibited a $45 \%$ relative reduction in overall mortality $v$. abstainers, well beyond the non-significant $31 \%$ relative reduction observed when only moderate alcohol intake was taken into account. This finding suggests that the assessment of an overall drinking pattern is better than the simple appraisal of the total amount of alcohol intake to capture the potential effect of alcohol consumption on mortality. Other aspects of the drinking pattern beyond total intake are able to provide additional relevant information.

The additional information gained by including further dimensions of alcohol consumption seems biologically sound because blood alcohol concentration depends, among other factors, on the quantity and frequency of alcohol consumption. Therefore, the same amount of total alcohol ingested will lead to higher blood alcohol concentration if it is consumed in a shorter period of time. Similarly, the same frequency of consumption will lead to higher blood alcohol concentration when the amount consumed in a single occasion is higher. Previous investigations have reported an increased risk of death for decreasing drinking-days ${ }^{(20,21)}$, or for the presence of heavy drinking occasions ${ }^{(22)}$, at the same level of alcohol consumption. Similar associations have been reported for myocardial infarction or major coronary events $^{(23,24)}$. Also, higher concentrations of alcohol in the gastrointestinal tract may have a local carcinogenic effect and higher blood alcohol concentrations may promote pancreatitis and liver disease ${ }^{(25)}$. These previous findings support the harmful effects of highly concentrated consumption patterns (binge drinking).

The type of beverage consumed is another important point. Although not all authors agree, moderate wine consumption has been associated with a lower risk of cardiovascular ${ }^{(26-31)}$, cancer $^{(26,28,29)}$, external-cause ${ }^{(26,28)}$ and all-cause mortality $^{(21,26-30,32-34)}$, and with longer life expectancy ${ }^{(31)}$ compared with beer or spirit consumption. Accordingly, we observed a lower rate of mortality among wine drinkers compared with non-wine drinkers. This protective benefit may be due in part to the quantity of alcohol consumed ${ }^{(35,36)}$, but it can specially be strengthened by the presence of considerable amounts of polyphenols in wine ${ }^{(37)}$. Moreover, wine consumption has previously been related to beneficial cardiovascular $^{(38-40)}$ and anti-carcinogenic effects ${ }^{(41,42)}$. An alternative explanation for this association is that wine drinkers are healthier than non-wine drinkers in other aspects ${ }^{(43)}$. For instance, dietary habits may differ from wine drinkers to beer drinkers or spirit drinkers. In order to account for confounding by these factors, several lifestyle variables, quality diet parameters and health indicators were included in the multipleadjusted models. In addition, some of the associations found in previous studies may be due to different patterns of consumption among wine drinkers than in other drinkers. 
Actually, preference for wine drinking has been associated with a less concentrated pattern of consumption ${ }^{(44)}$. Preferentially, wine drinkers are known to be at a lower risk of becoming heavy drinkers ${ }^{(45)}$. We found an inverse association between preference for wine and mortality, independently of other aspects of the alcohol-drinking pattern. On the contrary, preference for spirits did not show the same inverse association with the risk of mortality as wine. This differential effect also reported in a previous cohort study ${ }^{(31)}$ could be explained because spirits are distilled beverages with higher concentrations of alcohol per unit, but with lower concentrations of polyphenols. Wine drinkers are also known to be at a lower risk of being involved in violent deaths than beer or spirit drinkers ${ }^{(46)}$. This difference can also contribute to their lower mortality rates.

Alcohol intake was derived from a FFQ. This questionnaire inquired about dietary habits during the past year. However, alcohol intake includes some variability across different times of consumption. Therefore, other additional aspects of alcohol consumption were inquired in the baseline questionnaire, including the maximum number of drinks consumed in a weekday, during the weekend or in special occasions during the past year, and the pattern of drinking wine during meals. Participants drinking more than five drinks in a single occasion were at a higher risk of mortality. This is consistent with previous findings ${ }^{(47)}$, including also a recent research that found for binge drinkers a 14 to $168 \%$ increased risk of all-cause death among men and a 26 to $106 \%$ among women $^{(48)}$. Binge drinking has also been related to an increased risk of CHD and hypertension ${ }^{(49)}$, and of mortality $^{(50,51)}$. Other studies have reported a higher risk of death among heavy drinkers ${ }^{(22,52-54)}$, and these excess deaths are found to be especially attributable to cardiovascular and external causes ${ }^{(55)}$. Heavy drinking occasions may trigger atherosclerosis ${ }^{(56,57)}$ and increase arrhythmias ${ }^{(58)}$, sexually transmitted disease ${ }^{(59)}$, violence ${ }^{(60)}$ and traffic injuries.

Another important issue is the differential effect of consuming alcoholic beverages with meals or outside of meals. Some possible consequences of the consumption of alcohol with meals have been investigated: hypoglycaemic and insulinlowering effects ${ }^{(61)}$; reduced postprandial blood pressure among hypertensive patients ${ }^{(62)}$; or a reduction in LDL susceptibility to lipid peroxidation ${ }^{(63)}$. Only Trevisan et al. ${ }^{(64)}$ reported an increased risk of cardiovascular, cancer and all-cause mortality ( $51 \%$ increased risk) among drinkers outside of meals compared with drinkers of alcohol preferentially during meals, independently of the quantity of alcohol consumed. The present results are consistent with their findings.

Previous studies have frequently used the abstainers group as the reference category. However, artificially elevated rates of mortality in abstainers due to a higher mortality among former drinkers or to the avoidance of alcohol drinking because of medical causes ('sick quitter' hypothesis) may introduce some bias ${ }^{(65)}$. This was the reason why we did not use always the abstainers as the reference category. If another category is used as the reference, as we did in Tables 3 and 4, this potential bias will only affect the specific comparison for the abstainers group. 
Table 4. Mortality hazard ratios (HR) for each component of the Mediterranean alcohol-drinking pattern, using the best score (only among drinkers) as the reference category, in the Seguimiento Universidad de Navarra Project 1999-2012

(Hazard ratios and $95 \%$ confidence intervals)

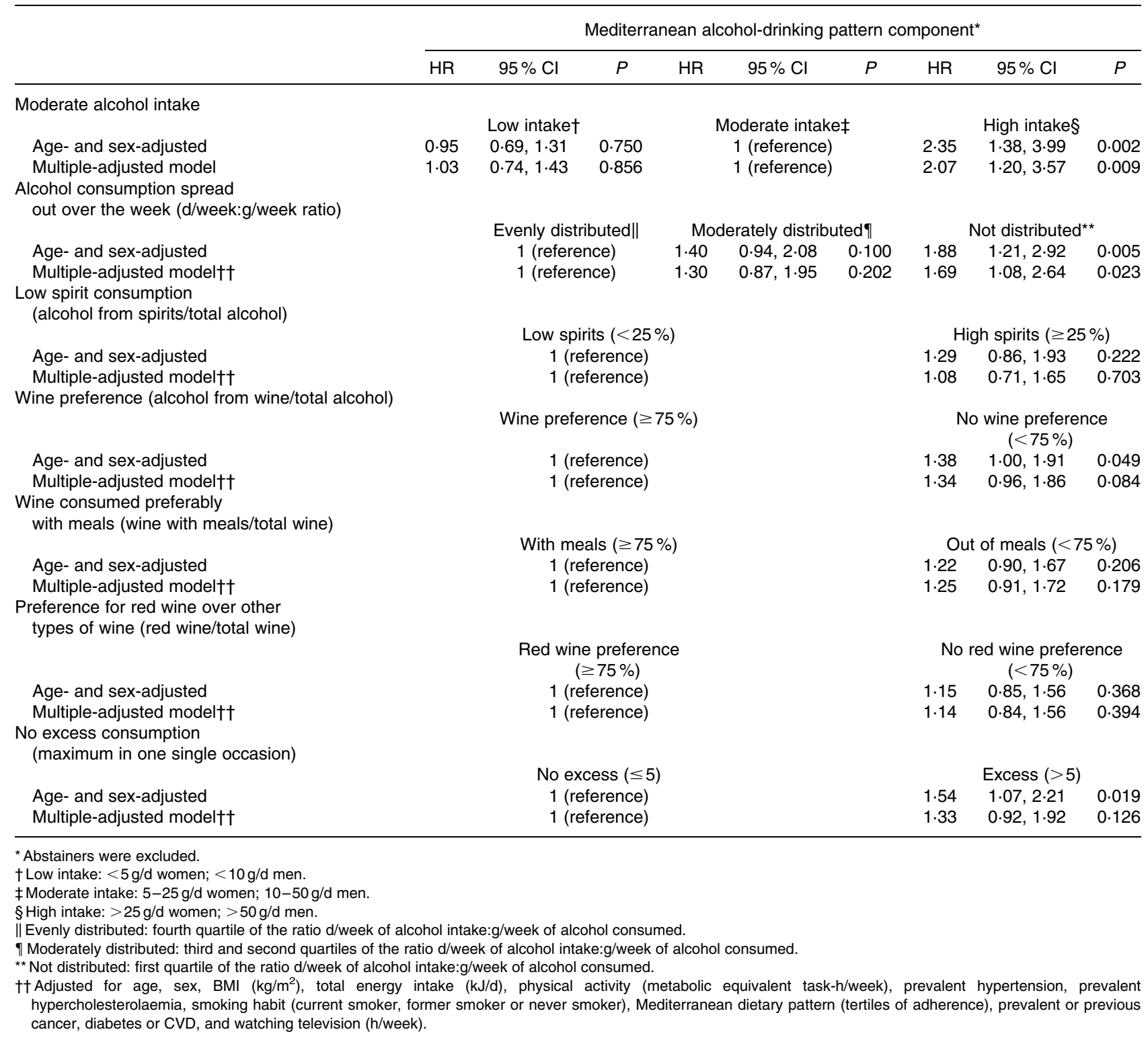

We did not adjust our analyses for socio-economic status. Participants in the SUN cohort study are all university graduates. Therefore, our sample is fairly homogeneous in this aspect, and this fact reduces the potential confounding by educational and socio-economic status. However, additional adjustment for educational levels did not substantially change the results.

Some degree of misclassification is possible in self-reported alcohol consumption and alcohol-drinking patterns. However, in the validation study of the $\mathrm{FFQ}^{(13)}$, the correlation coefficient for alcohol $(r$ 0.88) intake was higher than that for most other nutrients. Moreover, if there is some degree of misclassification, it will be more probably non-differential and therefore the association would be probably driven towards the null value.

The cut-off points for different items of the MADP intended to capture the concept of the traditional Mediterranean drinking pattern ${ }^{(10,15,16)}$. However, some of these cut-off points may be debatable and some degree of arbitrariness needs to be acknowledged as a limitation. In spite of that, the results of some sensitivity analyses after changing the cut-off points for different items showed the robustness of the association between the MADP score and total mortality.

Some items of the MADP could potentially exhibit collinearity. However, in our database, we observed $\kappa$ coefficients for every two items ranging from $-0 \cdot 103$ to $+0 \cdot 31$. Therefore, collinearity seems not to be a very relevant issue. Moreover, collinearity could be used to advantage because patterns are characterised on the basis of a comprehensive assessment of related behaviours ${ }^{(66)}$

Studies investigating one single aspect of alcohol consumption have some limitations: they may be confounded 
either by other aspects or by the whole pattern; the effect of a single aspect could be small and not detectable; the correlation between aspects makes it difficult to assess them separately ${ }^{(67)}$. Therefore, since drinking alcohol involves more dimensions than the specific amount of alcohol consumed, the best way to fully appraise the association between alcohol-drinking choices and health is to adopt the dietary pattern approach. This is currently the customary and accepted approach in other fields of nutritional epidemiology because this comprehensive approach allows for synergies and interactions, pre-empts confounding by other aspects of the pattern, gets closer to the real world and gives a more practical basis to issue public health recommendations ${ }^{(66)}$.

The limits for alcohol intake that we proposed might be seen as apparently high compared with some current guidelines. However, these guidelines usually do not take into account the role of the whole pattern of alcohol consumption.

In summary, the consumption of alcohol following a pattern of consumption that gets away from the traditional Mediterranean drinking habits is associated with a higher risk of mortality, as it is the total abstention of alcohol in comparison with good conformity to the traditional Mediterranean drinking pattern. Notwithstanding this result, perhaps the most sensible conclusion is that abstainers should not be counselled to start drinking because if they do it in a wrong way they may adopt drinking patterns with the potential to increase their risk of death. It is worth not to forget that alcohol intake is the eighth global death risk factor and the third risk factor for disease and disability measured in disability-adjusted life years ${ }^{(68)}$. However, if a person goes for drinking, the Mediterranean drinking pattern can be considered a sensible and healthy way of consuming alcohol.

\section{Acknowledgements}

We are indebted to the participants of the SUN Project for their continued cooperation and participation. We thank other members of the SUN Group: A. Alonso, F. J. Basterra-Gortari, S. Benito, J. de Irala, C. de la Fuente-Arrillaga, M. DelgadoRodríguez, F. Guillén-Grima, J. Krafka, J. Llorca, C. López del Burgo, A. Marti, J. A. Martínez, J. M. Nuñez-Córdoba, A. M. Pimenta, M. Ruiz-Canela, D. Sánchez, C. Sayón-Orea, M. Seguí-Gómez, M. Serrano-Martínez and Z. Vázquez.

The present study, as part of the SUN Project, was supported by Instituto de Salud Carlos III (PI01/0619, PI030678, PI040233, PI042241, PI050976, PI070240, PI070312, PI081943, PI080819, PI1002658, PI1002293, PI1300615, RD06/ 0045, 2010/087, G03/140 and Rio Hortega CM10/00072 to E. T.), the Navarra Regional Government (36/2001, 43/2002, $41 / 2005,45 / 2011$ and 36/2008), the University of Navarra (PIUNA 9923), CIBER Fisiopatología de la Obesidad y Nutrición (CIBERObn), Instituto de Salud Carlos III and Ministerio de Educación, Cultura y Deporte (A. G., FPU). The funders had no role in the design, analysis or writing of this article.

The authors' contributions are as follows: A. G. conducted the literature review, participated in the design of the present study, cleaned the data, conducted the main statistical analyses and prepared the first draft of the manuscript; M. B.-R.,
E. T., M. G.-L., J. J. B., R. E. and M. A. M.-G. participated in the statistical analysis plan and in the design of the present study, revised the manuscript and contributed to the interpretation of the findings; M. A. M.-G., being the guarantor, supervised all the steps in the statistical analyses and preparation of the manuscript. All authors critically revised the manuscript for important intellectual content and approved the final version.

R. E. reports serving on the board of and receiving lecture fees from the Research Foundation on Wine and Nutrition (FIVIN), serving on the boards of the Beer and Health Foundation and the European Foundation for Alcohol Research (ERAB), receiving lecture fees from Cerveceros de España and Sanofi-Aventis, and receiving grant support through his institution from Novartis, outside the submitted work. None of the other authors has any conflict of interest.

\section{References}

1. Costanzo S, di Castelnuovo A, Donati MB, et al. (2010) Alcohol consumption and mortality in patients with cardiovascular disease: a meta-analysis. J Am Coll Cardiol 55, 1339-1347.

2. Jin M, Cai S, Guo J, et al. (2013) Alcohol drinking and all cancer mortality: a meta-analysis. Ann Oncol 24, 807-816.

3. Di Castelnuovo A, Costanzo S, Bagnardi V, et al. (2006) Alcohol dosing and total mortality in men and women: an updated meta-analysis of 34 prospective studies. Arch Intern Med 166, 2437-2445.

4. Urbano-Marquez A, Estruch R, Navarro-Lopez F, et al. (1989) The effects of alcoholism on skeletal and cardiac muscle. $N$ Engl J Med 16, 409-415.

5. Fernandez-Sola J, Estruch R, Grau JM, et al. (1994) The relation of alcoholic myopathy to cardiomyopathy. Ann Intern Med 120, 529-536.

6. Corrao G, Bagnardi V, Zambon A, et al. (2004) A metaanalysis of alcohol consumption and the risk of 15 diseases. Prev Med 38, 613-619.

7. Rimm EB, Williams P, Fosher K, et al. (1999) Moderate alcohol intake and lower risk of coronary heart disease: meta-analysis of effects on lipids and haemostatic factors. BMJ 319, 1523-1528.

8. Estruch R, Sacanella E, Badia E, et al. (2004) Different effects of red wine and gin consumption on inflammatory biomarkers of atherosclerosis: a prospective randomized crossover trial. Effects of wine on inflammatory markers. Atherosclerosis 175, 117-123.

9. Facchini F, Chen YD \& Reaven GM (1994) Light-to-moderate alcohol intake is associated with enhanced insulin sensitivity. Diabetes Care 17, 115-119.

10. Heath DB (1997) Cultural variations among drinking patterns. In Drinking Patterns and their Consequences, pp. 103-128 [M Grant and J Litvak, editors]. Washington, DC: Taylor \& Francis.

11. Valencia-Martin JL, Galan I \& Rodriguez-Artalejo F (2007) Binge drinking in Madrid, Spain. Alcohol Clin Exp Res 31, 1723-1730.

12. Seguí-Gómez M, de la Fuente C, Vázquez Z, et al. (2006) Cohort profile: the 'Seguimiento Universidad de Navarra' (SUN) study. Int J Epidemiol 35, 1417-1422.

13. Martin-Moreno JM, Boyle P, Gorgojo L, et al. (1993) Development and validation of a food frequency questionnaire in Spain. Int J Epidemiol 22, 512-519.

14. Trichopoulou A, Costacou T, Bamia C, et al. (2003) Adherence to a Mediterranean diet and survival in a Greek population. $N$ Engl J Med 348, 2599-2608. 
15. Renaud S \& de Lorgeril M (1992) Wine, alcohol, platelets, and the French paradox for coronary heart disease. Lancet 339, 1523-1526.

16. Willett WC, Sacks F, Trichopoulou A, et al. (1995) Mediterranean diet pyramid: a cultural model for healthy eating. $A m \mathrm{~J}$ Clin Nutr 61, 1402S-1406S.

17. World Health Organization (2010) International classification of diseases, 10th revision. http://www.who.int/classifications/ icd/ICD10Volume2_en_2010.pdf

18. Martínez-González MA, López-Fontana C, Varo JJ, et al. (2005) Validation of the Spanish version of the physical activity questionnaire used in the Nurses' Health Study and the Health Professionals' Follow-up Study. Public Health Nutr 8, 920-927.

19. Trichopoulou A, Bamia C \& Trichopoulos D (2009) Anatomy of health effects of Mediterranean diet: Greek EPIC prospective cohort study. BMJ 338, b2337.

20. Tolstrup JS, Jensen MK, Tjønneland A, et al. (2004) Drinking pattern and mortality in middle-aged men and women. Addiction 99, 323-330.

21. Baglietto L, English DR, Hopper JL, et al. (2006) Average volume of alcohol consumed, type of beverage, drinking pattern and the risk of death from all causes. Alcohol Alcohol 41, 664-671.

22. Rehm J, Greenfield TK \& Rogers JD (2001) Average volume of alcohol consumption, patterns of drinking, and all-cause mortality: results from the US National Alcohol Survey. Am J Epidemiol 153, 64-71.

23. Mukamal KJ, Conigrave KM, Mittleman MA, et al. (2003) Roles of drinking pattern and type of alcohol consumed in coronary heart disease in men. N Engl J Med 348, 109-118.

24. McElduff P \& Dobson AJ (1997) How much alcohol and how often? Population based case-control study of alcohol consumption and risk of a major coronary event. BMJ $\mathbf{3 1 4}$, $1159-1164$

25. Wetterling T, Veltrup C, Driessen M, et al. (1999) Drinking pattern and alcohol-related medical disorders. Alcohol Alcohol 34, 330-336.

26. Renaud SC, Guéguen R, Siest G, et al. (1999) Wine, beer, and mortality in middle-aged men from eastern France. Arch Intern Med 159, 1865-1870.

27. Harriss LR, English DR, Hopper JL, et al. (2007) Alcohol consumption and cardiovascular mortality accounting for possible misclassification of intake: 11-year follow-up of the Melbourne Collaborative Cohort Study. Addiction 102, $1574-1585$.

28. Klatsky AL, Friedman GD, Armstrong MA, et al. (2003) Wine, liquor, beer, and mortality. Am J Epidemiol 158, 585-595.

29. Grønbaek M, Becker U, Johansen D, et al. (2000) Type of alcohol consumed and mortality from all causes, coronary heart disease, and cancer. Ann Intern Med 133, 411-419.

30. Theobald H, Bygren LO, Carstensen J, et al. (2000) A moderate intake of wine is associated with reduced total mortality and reduced mortality from cardiovascular disease. $J$ Stud Alcohol 61, 652-656.

31. Streppel MT, Ocké MC, Boshuizen HC, et al. (2009) Longterm wine consumption is related to cardiovascular mortality and life expectancy independently of moderate alcohol intake: the Zutphen Study. J Epidemiol Community Health 63, 534-540.

32. Nielsen NR, Schnohr P, Jensen G, et al. (2004) Is the relationship between type of alcohol and mortality influenced by socio-economic status? J Intern Med 255, 280-288.

33. Grønbaek M, Deis A, Sørensen TI, et al. (1995) Mortality associated with moderate intakes of wine, beer, or spirits. BMJ 310, 1165-1169.
34. Strandberg TE, Strandberg AY, Salomaa VV, et al. (2007) Alcoholic beverage preference, 29-year mortality, and quality of life in men in old age. J Gerontol A Biol Sci Med Sci 62, 213-218.

35. Vlachopoulos C, Tsekoura D, Tsiamis E, et al. (2003) Effect of alcohol on endothelial function in healthy subjects. Vasc Med 8, 263-265.

36. Shai I, Rimm EB, Schulze MB, et al. (2004) Moderate alcohol intake and markers of inflammation and endothelial dysfunction among diabetic men. Diabetologia 47, 1760-1767.

37. Arranz S, Chiva-Blanch G, Valderas-Martinez P, et al. (2012) Wine, beer, alcohol and polyphenols on cardiovascular disease and cancer. Nutrients 4, 759-781.

38. Kiviniemi TO, Saraste A, Toikka JO, et al. (2007) A moderate dose of red wine, but not de-alcoholized red wine increases coronary flow reserve. Atherosclerosis 195, e176-e181.

39. Frankel EN, Kanner J, German JB, et al. (1993) Inhibition of oxidation of human low-density lipoprotein by phenolic substances in red wine. Lancet 341, 454-457.

40. Pace-Asciak CR, Hahn S, Diamandis EP, et al. (1995) The red wine phenolics trans-resveratrol and quercetin block human platelet aggregation and eicosanoid synthesis: implications for protection against coronary heart disease. Clin Chim Acta 235, 207-219.

41. Jang M, Cai L, Udeani GO, et al. (1997) Cancer chemopreventive activity of resveratrol, a natural product derived from grapes. Science 275, 218-220.

42. Prescott E, Grønbaek M, Becker U, et al. (1999) Alcohol intake and the risk of lung cancer: influence of type of alcoholic beverage. Am J Epidemiol 149, 463-470.

43. Mortensen EL, Jensen HH, Sanders SA, et al. (2001) Better psychological functioning and higher social status may largely explain the apparent health benefits of wine: a study of wine and beer drinking in young Danish adults. Arch Intern Med 161, 1844-1848.

44. Grønbaek M, Tjønneland A, Johansen D, et al. (2000) Type of alcohol and drinking pattern in 56,970 Danish men and women. Eur J Clin Nutr 54, 174-176.

45. Jensen MK, Andersen AT, Sørensen TI, et al. (2002) Alcoholic beverage preference and risk of becoming a heavy drinker. Epidemiology 13, 127-132.

46. Norström T (1998) Effects on criminal violence of different beverage types and private and public drinking. Addiction 93, 689-699.

47. Kauhanen J, Kaplan GA, Goldberg DE, et al. (1997) Beer binging and mortality: results from the Kuopio ischaemic heart disease risk factor study, a prospective population based study. BMJ 315, 846-851.

48. Graff-Iversen S, Jansen MD, Hoff DA, et al. (2013) Divergent associations of drinking frequency and binge consumption of alcohol with mortality within the same cohort. J Epidemiol Community Health 67, 350-357.

49. Murray RP, Connett JE, Tyas SL, et al. (2002) Alcohol volume, drinking pattern, and cardiovascular disease morbidity and mortality: is there a U-shaped function? Am J Epidemiol 155, 242-248.

50. Mukamal KJ, Maclure M, Muller JE, et al. (2005) Binge drinking and mortality after acute myocardial infarction. Circulation 112, 3839-3845.

51. Roerecke M, Greenfield TK, Kerr WC, et al. (2011) Heavy drinking occasions in relation to ischaemic heart disease mortality - an 11-22 year follow-up of the 1984 and 1995 US National Alcohol Surveys. Int J Epidemiol 40, 1401-1410.

52. Malyutina S, Bobak M, Kurilovitch S, et al. (2002) Relation between heavy and binge drinking and all-cause 
and cardiovascular mortality in Novosibirsk, Russia: a prospective cohort study. Lancet 360, 1448-1454.

53. Mørch LS, Johansen D, Løkkegaard E, et al. (2008) Drinking pattern and mortality in Danish nurses. Eur J Clin Nutr 62 , $817-822$

54. Kauhanen J, Kaplan GA, Goldberg DD, et al. (1997) Frequent hangovers and cardiovascular mortality in middle-aged men. Epidemiology 8, 310-314.

55. Laatikainen T, Manninen L, Poikolainen K, et al. (2003) Increased mortality related to heavy alcohol intake pattern. J Epidemiol Community Health 57, 379-384.

56. Gruchow HW, Hoffmann RG, Anderson AJ, et al. (1982) Effects of drinking patterns on the relationship between alcohol an coronary occlusion. Atherosclerosis 43, 393-404.

57. Kiechl S, Willeit J, Rungger G, et al. (1998) Alcohol consumption and atherosclerosis: what is the relation? prospective results from the Bruneck Study. Stroke 29, 900-907.

58. McKee M \& Britton A (1998) The positive relationship between alcohol and heart disease in eastern Europe: potential physiological mechanisms. $J$ R Soc Med 91, 402-407.

59. Standerwick K, Davies C, Tucker L, et al. (2007) Binge drinking, sexual behaviour and sexually transmitted infection in the UK. Int J STD AIDS 18, 810-813.

60. Brewer RD \& Swahn MH (2005) Binge drinking and violence. JAMA 294, 616-618.
61. Hätönen KA, Virtamo J, Eriksson JG, et al. (2012) Modifying effects of alcohol on the postprandial glucose and insulin responses in healthy subjects. Am J Clin Nutr 96, 44-49.

62. Foppa M, Fuchs FD, Preissler L, et al. (2002) Red wine with the noon meal lowers post-meal blood pressure: a randomized trial in centrally obese, hypertensive patients. $J$ Stud Alcohol 63, 247-251.

63. Fuhrman B, Lavy A \& Aviram M (1995) Consumption of red wine with meals reduces the susceptibility of human plasma and low-density lipoprotein to lipid peroxidation. Am J Clin Nutr 61, 549-554.

64. Trevisan M, Schisterman E, Mennotti A, et al. (2001) Drinking pattern and mortality: the Italian Risk Factor and Life Expectancy pooling project. Ann Epidemiol 11, 312-319.

65. Shaper AG, Wannamethee G \& Walker M (1988) Alcohol and mortality in British men: explaining the U-shaped curve. Lancet 2, 1267-1273.

66. Hu FB (2002) Dietary pattern analysis: a new direction in nutritional epidemiology. Curr Opin Lipidol 13, 3-9.

67. Schulze MB \& Hoffmann K (2006) Methodological approaches to study dietary patterns in relation to risk of coronary heart disease and stroke. Br J Nutr 95, 860-869.

68. World Health Organization (2011) Global Status Report on Alcohol and Health. Geneva: WHO. http://www.who. int/substance_abuse/publications/global_alcohol_report/ msbgsruprofiles.pdf 